\title{
Meeting Report \\ Developing a Road Map to Spread Genomic Knowledge in Africa: 10th Conference of the African Society of Human Genetics, Cairo, Egypt
}

\begin{abstract}
Ghada Y. El-Kamah, ${ }^{1 \star}$ Amal M. Mohamed, ${ }_{7}^{1}$ Yehia Z. Gad, ${ }^{1}$ Sonia Abdelhak, ${ }^{2}$ Branwen J. Hennig, ${ }^{3}$ Raj S. Ramesar, ${ }^{4}$ Guida Landouré, ${ }^{5,6}$ Amadou Gaye, ${ }^{7}$ Melanie J. Newport, ${ }^{8}$ Scott M. Williams, ${ }^{9}$ and Michèle Ramsay ${ }^{10}$

${ }^{1}$ Human Genetics and Genome Research Division, Centre of Scientific Excellence of Human Genetics, National Research Centre, Cairo, Egypt; ${ }^{2}$ Laboratory of Biomedical Genomics and Oncogenetics, Institut Pasteur de Tunis, University of Tunis El Manar, Tunis, Tunisia; ${ }^{3}$ Science Division, Wellcome, London, United Kingdom; ${ }^{4}$ Division of Human Genetics, Department of Pathology, Faculty of Health Science, University of Cape Town and Affiliated Hospitals, Cape Town, South Africa; ${ }^{5}$ Faculté de Médecine et d'Odontostomatologie, Université des Sciences, des Techniques et des Technologies de Bamako, Bamako, Mali; ${ }^{6}$ Service de Neurologie, Centre Hospitalier Universitaire du Point G, Bamako, Mali; ${ }^{7}$ National Institutes of Health, National Human Genome Research, Bethesda, Maryland; ${ }^{8}$ Brighton and Sussex Centre for Global Health Research, Brighton and Sussex Medical School, University of Sussex, Falmer, United Kingdom; ${ }^{9}$ Department of Population and Quantitative Health Sciences, Institute of Computational Biology, Case Western Reserve University, Cleveland, Ohio; ${ }^{10}$ Sydney Brenner Institute for Molecular Bioscience, Faculty of Health Sciences, University of the Witwatersrand, Johannesburg, South Africa
\end{abstract}

\begin{abstract}
The tenth conference of the African Society of Human Genetics was held in Egypt with the theme "Human Genetics and Genomics in Africa: Challenges for Both Rare and Common Genetic Disorders." Current research was presented, and we discussed visions for the future of genomic research on the African continent. In this report, we summarize the presented scientific research within and relevant to Africa as presented by both African and non-African scientists. We also discuss the current situation concerning genomic medicine and genomic research within the continent, difficulties in implementing genetic services and genomic medicine in Africa, and a road map to overcome those difficulties and meet the needs of the African researchers and patients.
\end{abstract}

\section{INTRODUCTION}

The African Society of Human Genetics (AfSHG), launched in 2003, aims to propose strategies for the translation of genomic knowledge to address the public health burden of diseases across the continent and to develop a road map for increasing and spreading this knowledge within and beyond the continent. ${ }^{1}$ The AfSHG proposed new strategies ${ }^{2,3}$ to provide assistance to countries with the highest burden of genetic disorders and ensure that Africa is not left behind in a "genomic divide." As a result, several funding bodies have invested in genomic research that has contributed to building research capacity in Africa. A key instrument of this investment has been the Human Heredity and Health in African Consortium (H3Africa), a Pan-African research consortium focusing on human health-related genomics and genetics research on the continent, funded largely by the NIH in the United States and the Wellcome Trust in the United Kingdom. This initiative was first discussed as a possibility 10 years earlier at the 2007 AfSHG meeting in Cairo. The H3Africa fostered the beginning of large-scale implementation of genomic technologies aimed at improving the health of Africans (http://h3africa.org) during a first phase (2012 to 2017) that included eight collaborative centers, seven research projects, six ethics projects, three H3Africa biorepository sites, and a Pan-African bioinformatics network with 26 research groups in 27 African countries. ${ }^{4-9}$ Enhanced research capacity requires appropriate planning and funding to develop thriving and sustainable networks throughout the continent.

In line with these goals, ${ }^{3-8,10,11}$ the tenth conference of the AfSHG was held in partnership with the National Society of

\footnotetext{
*Address correspondence to Ghada Y. El-Kamah, Human Genetics and Genome Research Division, Centre of Scientific Excellence of Human Genetics, NRC, 24 Ghaza Street, Mohandesin 12411, Cairo,
} Egypt. E-mail: ghadaelkamah@otmail.com
Human Genetics in Egypt and the H3Africa Consortium in Cairo from November 16 to 18, 2017. Four hundred fiftythree scientists from 32 countries attended the meeting (Figure 1). To encourage attendance by investigators and trainees with limited resources, 72 travel fellowships to junior and senior African researchers were granted. In an attempt to ensure unity across the African continent, simultaneous translation into French was provided. Webcasting of many of the conference's sessions were available to allow broader participation (http://www.nshg-society.eg.net/ activities.html).

Satellite Events. Young Researchers' Forum. A Young Researchers' Forum (YRF) was held the day before the main meeting so that young researchers could present their research and exchange experiences. There were 16 oral and 33 poster presentations, and presenters interacted with their peers and senior researchers. The theme was "Hand in Hand to Change the Genetic Research Future over the African Land." Presented scientific research topics included common traits with genetic susceptibility, genetic diagnostics, and cancer, and six young researchers were awarded special prizes: three to the best oral and three to the best poster presenters in the form of travel grants for the next AfSHG conference. Other awardees received books. An important outcome of these YRFs at the AfSHG meetings is the development of formal and informal networks to enhance relationships and develop future partnerships among emerging researchers.

Educational initiative. An "early bird" workshop on nextgeneration sequencing (NGS) was run by Prof. Samar Kassim and Dr. Amadou Gaye over three mornings that covered the basics of NGS library preparation, data processing pipeline, and quality control and provided exemplars of single-variant and gene-based analyses. This session was instrumental in exposing African researchers to the latest genomic technologies, data analyses, and their uses. 


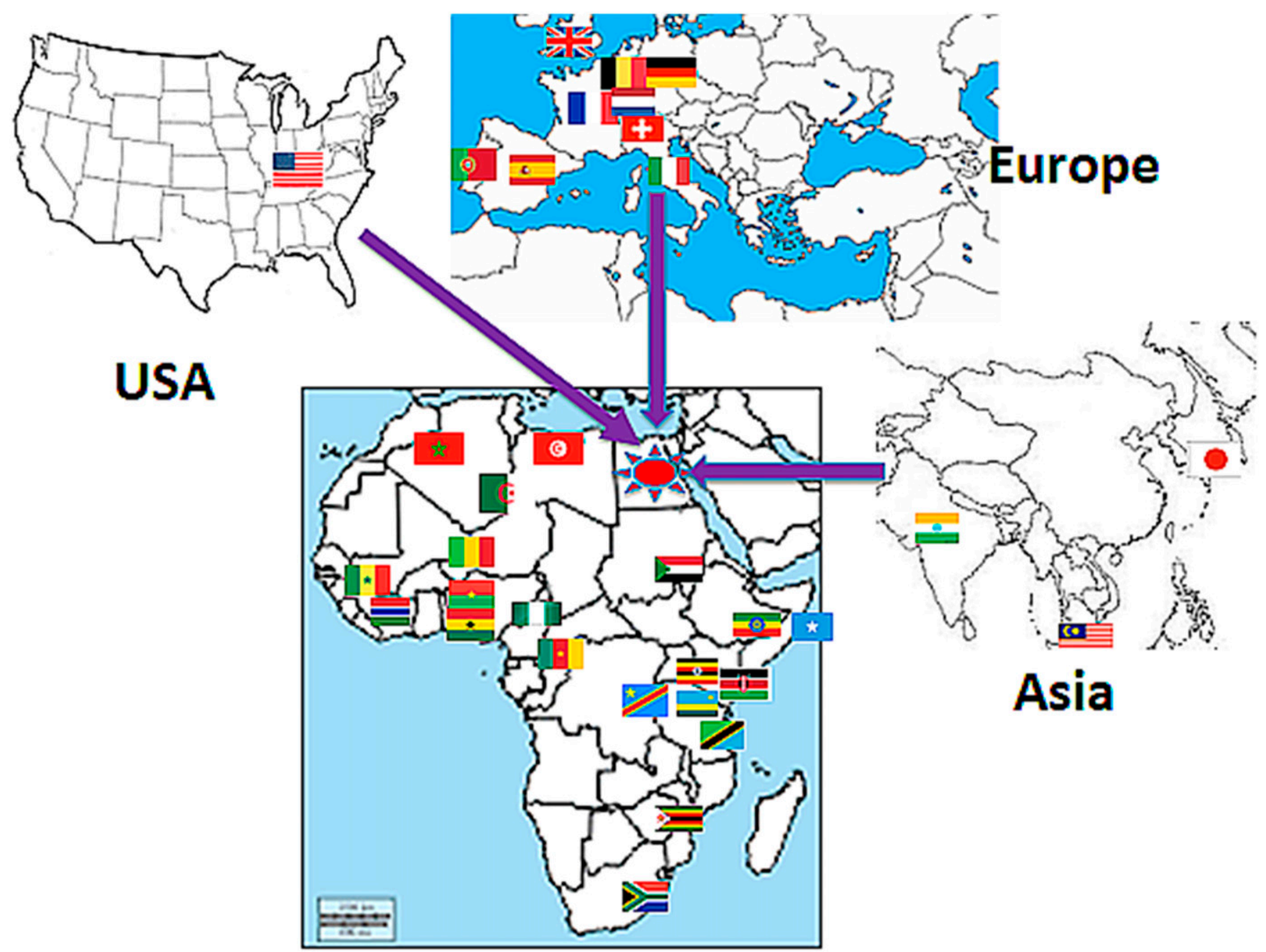

FIGURE 1. Participant countries at the 10th African Society of Human Genetics meeting, Cairo, 2017. This figure appears in color at www.ajtmh.org.

\section{SCIENTIFIC HIGHLIGHTS FROM THE MAIN CONFERENCE}

The official opening of the conference included introductions by Michèle Ramsay (University of the Witwatersrand, South Africa), president of the AfSHG, Samia Temtamy, president of the Egyptian National Society of Human Genetics, and Ambroise Wonkam (University of Cape Town, South Africa) from the steering committee of the H3Africa. In addition, the Egyptian assistant foreign minister for African affairs (Mohamed Idris), vice president of the National Research Centre, Egypt (Mohamed Hashem), and president of the Academy of Scientific Research and Technology, Egypt (Mahmoud Sakr), addressed the meeting.

The meeting began with an appraisal of African genomic research, discussing the future impact of genetic diversity and African genomic data on understanding and improving the efficacy and safety of therapeutic drugs on the path to attaining individualized medicine (precision medicine). In light of many of these issues, the first plenary talk at the conference by Charles Rotimi, founding president of the AfSHG and head of the Center for Research on Genomics and Global Health (National Genome Research Institute, USA), addressed the impact of Africa's genetic diversity in the dawn of precision medicine. In his presentation, he challenged African scientists with a new dream to develop a five-million Pan-African population cohort, one million from each African region, as a resource for studying disease prevalence and causality and to provide a framework for human genetics on the continent. The information from such a cohort would propel research into precision medicine within Africa and beyond.

Next, Yehia Gad (National Research Center, Egypt), a senior medical geneticist, brought a unique Egyptian flavor to the meeting with a presentation on genomic research to elucidate the identity of a number of suspected royal Egyptian mummies, investigating their family relationships to historically identified mummies and making use of genetic kingship analysis. Providing an insight into the state of medical genetics and diagnostics in Africa, Prosper Lukusa, president of the Congolese Society for Human Genetics, discussed hardships in establishing genetic testing in the Democratic Republic of Congo the dire need for support. He proposed sharing existing resources with other African and/or European laboratories. Rokhaya Ndiaye (Cheikh Anta Diop University, Dakar, Senegal) reported on a pilot project to investigate the incidence of congenital malformations in Dakar. Researchers 
from Cameroon and Egypt discussed their initial results from NGS of gene panels and whole exome analyses that potentially led to the identification of the causal mutations for genetic disorders. The H3Africa Consortium announced the design of a $\sim 2$.3-million single nucleotide variant genotyping array as an African-centric, unbiased, and cost effective solution for genome-wide analysis for complex traits and population genetics research on the continent.

A session on "Stem cells in medical genetics" discussed the capability of dental pulp stem cells to promote peripheral and central nerve regeneration as well as vasculogenesis/ angiogenesis in the ischemic hind limb (Misako Nakashima, Nagoya University, Japan, and Shin Kwamata, Foundation for Biomedical Research and Innovation Center for Cell Therapy, Japan). Secretome-based therapy for Alzheimer's disease was presented (Misako Nakashima). Stem cell research targeting diabetes therapy with regard to potential deriving functional human pancreatic $\beta$ cells in vitro and $\beta$ cell transplantation therapy in disease modeling was also presented (Reham Aly, National Research Centre, Egypt).

The Egyptian maxillofacial dental genetics team reported on combining stem cell therapy with autogenous growth factors in treatments involving bone, soft tissue, fat, and muscle regeneration for different clinical cases that resulted in extraordinary recovery (Ahmad Farouk, National Research Centre, Egypt, and Nermeen Ahmad, National Research Centre, Egypt). Another Egyptian study reported stem cells obtained from the periodontal ligament as a promising treatment modality for regenerating tissues destroyed by the periodontal diseases (Maha Abdelfattah, National Research Centre, Egypt).

Talks in the session on cancer genetics and genomics included new methods for screening for high-risk human papilloma virus strains by testing for the E6/E7 oncoprotein reported in Rwanda (Leon Mutesa, University of Rwanda). Scott Williams (Case Western Reserve University, USA) spoke about cancer and coevolution with Helicobacter pylori concluding that the interaction between the host and pathogens, in terms of ancestry, could account for a large proportion of difference in disease risk previously associated with geography. In particular, African $H$. pylori ancestry that was relatively benign in people of African descent was shown to be deleterious in individuals with substantial Native American ancestry. Collaborative work in epidemiological genetic studies and clinical genomics, with the aim of primary prevention and early detection programs of cancer, was discussed while reporting an increased incidence of cancer, particularly hepatocellular carcinoma in Africa (Abdel Hady). There were also studies pointing to a central role of the Fanconi anemia pathway in cellular and chromosomal resistance to DNA damage in melanoma cells (Filippo Rosselli, Gustave Roussy Institute, Paris, France).

Genomic variation was explored, specifically with regard to African-descent population genetic diversity and its impact on health and variable distribution of different disorders throughout the sessions on cancer, other common noncommunicable genetic disorders, and infectious diseases. There was an emphasis on gene-environment interactions and their impact on the distribution of common and complex diseases in Africa (Ayo Doumatey, National Human Genome Research Institute (NHGRI, USA) and Raj Ramesar, University of Cape Town, South Africa).
In the field of genetics of rare disorders and epidemiology, a study on genodermatoses in Egypt presented an example of the increased incidence of disorders in African countries where consanguineous marriages are common (Ghada El Kamah, National Research Center, Egypt and Mohamed Abdelkader, National Research Centre, Egypt). Whole-exome sequencing in consanguineous families with genetic disorders was reported as an effective tool for novel disease-gene discovery and for the detection of novel mutations in previously identified genes causing disease in patients from Tunisia, Egypt, and Mali (Sonia Abdelhak, Research Laboratory of Biomedical Genomics and Oncogenetics, Istitut Pasteur, Tunisia; Inas Mazen, National Research Centre, Egypt; and Guida Landoure, Faculté de Médecine et d'Odontostomatologie, USTTB and Service de Neurologie, Centre Hospitalier Universitaire du Point G, Mali).

The clinical and molecular landscape of neurogenetics, including brain malformations, microcephaly, and intracranial calcifications, were presented (Ghada Abdel-Salam, National Research Centre, Egypt, and Maha Zaki, National Research Centre, Egypt) together with some solutions such as 3D surgery in the treatment of unicoronal craniosynostosis (Tarek Elbanouby, Al-Azhar University, Egypt). An H3Africa study reported an initiative to discover causative genes for neurogenetic disorders in African communities, with high fertility and consanguinity rates. Revising the content of gene panels, to avoid erroneous conclusions in African populations, was an important recommendation from the meeting. This followed reports on studies showing that NGS combined with linkage analysis, whole-exome sequencing, and whole-genome sequencing facilitated novel disease gene discovery (Faisal Fadlelmola, University of Khartoum, Sudan). Kenneth Fischbeck (National Institute of Neurological Disorders and Stroke, USA) presented studies on neurological diseases and provided perspectives on treatment, some of which are already in the market or in Phase III clinical trials.

During the session on NGS approaches and bioinformatics training, there were presentations on big data and genomics, variability in allele frequencies, linkage disequilibrium, genomewide association studies (Hanno Boltz, University Hospital of Cologne, Germany), current and prospective use of bioinformatics to serve genetics, and genomic research in Africa. Benefits, challenges, and pitfalls of decoding inherited diseases in consanguineous families in the era of high-throughput DNA sequencing were discussed. H3Africa's bioinformatics network, H3ABioNet [https://h3abionet.org/], which has representation across countries from Cape Town, South Africa, to Cairo, Egypt, described the use of webinars as a model for providing a forum for consortium members working in bioinformatics and computational genomics as well as their application to various aspects of genomic medicine (Samar Kassim, Ain Shams University, Egypt).

Hans Hagen (Institute Pasteur, Paris) discussed the PanAfrican Coalition for Training in Research and Public Health that aims to provide collaborative and disciplinary platforms for training in both trusted and novel technologies across a range of disease domains, and the creation of a network allowing the sharing of skills and resources.

In the domain of translational genomics in African research, pharmacogenetics and diagnostic applications of genomic research in Africa were thoroughly discussed (Collen Masimirembwa, African Institute of Biomedical Science and 
Technology, Zimbabwe). The use of international collaborative databases, with consideration of different ethnic backgrounds, allows comparison of individuals suffering from a variety of genetic syndromes. This included reporting of both subjective clinical findings and objective facial recognition technology differences detected between various populations for selected syndromes (Maximilian Muenke, National Human Genome Research Institute, USA).

Obesity as one of the common traits with genetic susceptibility and contemporary health burden was discussed in light of concerns about the effect of obesity on transcriptomic changes and the use of multiple molecular tools to identify molecular changes associated with obesity and obesity-related traits; functionally, these can include epigenetic modifications, including miRNA regulation. Array-comparative genomic hybridization was presented as a tool for genetic analysis of changes in schizophrenic patients.

Hereditary disorders of the blood impose an important health burden in Africa, especially sickle cell anemia and the diagnosis of congenital hemolytic anemia. In the NGS era, a panel covering 26 genes was developed to address the problem of genetically heterogeneous undiagnosed congenital hemolytic anemia (Ambroise Wonkam and Celeste Bento). Mutagenesis in adaptive immunity addressed the genetic and biochemical mechanisms that showed that somatic hypermutations are diverted from their normal functions, being used in rather atypical ways to favor mutagenesis over accuracy (Said Aoufouchi, Gustave Roussy Cancer Center, France). Another study concerned with the analysis of DNA-protein cross-link damage induced by genotoxic agents concluded how living organisms cope with the threat from genotoxic agents and maintain their global genetic integrity (Amir Salem, National Research Centre, Egypt).

Noncoding RNAs, as new diagnostic markers, were presented as promising biomarkers that will be able to predict and enable early diagnosis of hepatocellular carcinoma patients (Olfat Shaker, Cairo University, Egypt). Studies on how severe dysfunction of the immune system affects clinical presentation of inborn errors of immunity and human diseases; how they are currently studied as a model to decipher immune mechanisms involved in the emergence of other associated clinical manifestations, including allergy, autoimmunity, and malignant lymphoproliferation; and how their genetic basis is shaping the understanding of those more complex multiple issues were discussed (Ridha Barbouche, University of Tunis, Tunisia).

The last session reported on establishing a center of excellence for human genetics in Egypt. Five clinical researchers highlighted the value and complementarity of coordinating different genetics subspecialties to better serve patients with genetic disorders and promote research (Khalda Amr, Mona Aglan, Mona Essawy, Naglaa Kholousy, and Khaled Gaber, all from the National Research Centre, Egypt).

Among important topics discussed, those affecting scientific research in an African setting were ethics and biobanking (Paul Ndebele, Medical Research Council of Zimbabwe), where ethical sufficiency of informed consent for justifying research has been important for the development of research resources. Challenges involving implementation and sustainability of African biobanks were discussed, stressing the quality of informed consent, particularly where possible future use of biological material is concerned. Participants from
Nigeria, Uganda, and Zimbabwe presented their experiences with biobanking. Under the umbrella of the H3Africa Consortium, there were discussions on the setup of well-coordinated, high quality biorepositories and the framework for evaluating the ethical soundness of genomic research and biobanking by ethics review boards across the African continent. What was evident is the shift in thinking with regard to "genomic sovereignty and exceptionalism" to recognizing the true value of sharing data and biological material-if there is any hope of the benefits of research being brought back to source research populations. However, what was also clear is that there are very different levels of engagement between funders and collaborators on the international stage, leading to the need for a complex series of discussions for engagement between researchers, communities, and governments (Ananyo Choudhury, University of the Witwatersrand; James Onyemata, Institute of Human Virology, South Africa; and Bazondile Dube-Marimbe, University of Zimbabwe).

There was a parallel workshop affiliated to the Human Variome Project concerned with hemoglobinopathies as a health problem on the continent and the impact of genomic data sharing on research (Zilfalil Bin Alwi, Hospital University Sains, Malaysia; Raj Ramesar, University of Cape Town; and NarazahYusoff, Hospital University Sains, Malaysia).

\section{STRATEGIC PLANNING FOR THE FUTURE}

The meeting planned to amplify research activities, enhance resources in Africa, and leverage synergies between the AfSHG, H3Africa, and other relevant initiatives, as well as to better engage African citizens in genomics. A key question is how the AfSHG can benefit those working on African genetics and achieve a sustainability model with a more diverse and stable funding. The following suggestions emanated from the meeting: 1) conduct live webinars to discuss common genetic issues, 2) mentor junior researchers to organize the discussion, 3) strengthen partnerships, and 4) organize future conferences in conjunction with relevant meetings for crossfertilization of research across disciplines.

\section{MAIN CONCLUSIONS FROM THE CONFERENCE}

There is an urgent need to leverage synergies and develop guidelines to achieve specific goals. We need to expand our efforts to close the genomic gap between Africa and other regions. The field of genetics is a rapidly expanding global initiative, and advancing genetics and technological knowledge in Africa depends on Pan-African as well as African-international cooperative arrangements between professionals. An excellent strategy would be to reinforce existing genetics centers that have a critical mass of trained people. The AfSHG is at a point where it needs to think carefully about the future and to understand what young scientists want from their society and how to fulfill their dreams and support their scientific ambitions in the years to come. A lack of local opportunities leads to Africa's loss of some 20,000 professionals to high-income countries every year. ${ }^{10}$ To move forward, it is essential to examine existing capacities, for instance, through building human genetics centers and research networks and/or hubs across the continent. This can be achieved through the development of 1) clinical phenotyping centers, 2) molecular phenotyping centers, 3) 
genotyping and sequencing capability, 4) data centers, and 5) sustainable biorepositories in Africa. ${ }^{3}$ Genetic diagnosis and application of genetic testing for rare and common genetic disorders should be the basis for future development. There is also a need to support training programs for clinical genetics, laboratory genetic testing, and genomics research. Seeking international support is mandatory for bridging the gaps in financial and technological resources in medical genetics practice in Africa to fulfill both recommendations. Another way to develop knowledge in genetics and genomic science through the existing networks is to work toward establishing an African school of human genetics.

The AfSHG presented ideas for a road map and a vision for sustaining and spreading genomic knowledge in Africa. African initiatives need acknowledgment of human genetics as significant to public health. The International Monetary Fund predicts Africa as the world's second fastest growingeconomy by $2020^{10-12}$ and trained, competitive scientists are essential to accelerate acquisition of knowledge and development across the continent. Skilled workforces can attract private-sector investment, and sustainability needs funding from Africa itself rather than relying only on foreign investors. It is difficult to define the scale of the challenges ahead, but there are clear and exciting opportunities for genetics and genomics on the continent.

Received May 31, 2019. Accepted for publication December 4, 2019.

Published online March 2, 2020.

Note: Supplemental information appears at www.ajtmh.org.

Acknowledgment: The American Society of Tropical Medicine and Hygiene (ASTMH) assisted with publication expenses.

Disclosure: B. J. H. reports that he is employed by the Wellcome Trust, a research funder based in the United Kingdom; however, no Wellcome funds have contributed to this publication.

Authors' addresses: Ghada Y. El-Kamah, Amal M. Mohamed, and Yehia Z. Gad, Human Genetics and Genome Research Division, Centre of Scientific Excellence of Human Genetics, NRC, Cairo, Egypt, E-mails: ghadaelkamah@hotmail.com, amalmahmoud15@ yahoo.com, and yehiagad@gmail.com. Sonia Abdelhak, Laboratory of Biomedical Genomics and Oncogenetics, Institut Pasteur de Tunis, University of Tunis El Manar, Tunis, Tunisia, E-mail: sonia.abdelhak@ gmail.com. Branwen J. Hennig, Science Division, Wellcome, London, United Kingdo, E-mail: b.hennig@wellcome.ac.uk. Raj S. Ramesar, Division of Human Genetics, Department of Pathology, Faculty of Health Science, University of Cape Town and Affiliated Hospitals, Cape Town, South Africa, E-mail: raj.ramesar@uct.ac.za. Guida Landouré, Faculté de Médecine et d'Odontostomatologie, USTTB, Bamako, Mali, and Service de Neurologie, Centre Hospitalier
Universitaire du Point G, Bamako, Mali, E-mail: glandoure@ gmail.com. Amadou Gaye, National Institutes of Health, National Human Genome Research, Bethesda, MD, E-mail: amadou.gaye@ nih.gov. Melanie J. Newport, Brighton and Sussex Centre for Global Health Research, Brighton and Sussex Medical School, University of Sussex, Falmer, United Kingdom, E-mail: m.j.newport@bsms.ac.uk. Scott M. Williams, Department of Population and Quantitative Health Sciences, Institute of Computational Biology, Case Western Reserve University, Cleveland, OH, E-mail: smw154@case.edu. Michèle Ramsay, Sydney Brenner Institute for Molecular Bioscience, Faculty of Health Sciences, University of the Witwatersrand, Johannesburg, South Africa, E-mail: michele.ramsay@wits.ac.za.

\section{REFERENCES}

1. Rotimi CN, 2004. Inauguration of the African Society of Human Genetics. Nat Genet 36: 544.

2. March K, 2016. How Africa Can Close its Continent-wide Science Funding Gap. Available at: https://theconversation.com/howafrica-can-close-its-continent-wide-science. funding-gap-55957. Accessed April 12, 2016.

3. Wonkam A, Muna W, Ramesar R, Rotimi CN, Newport MJ, 2010. Capacity-building in human genetics for developing countries: initiatives and perspectives in sub-Saharan Africa. Public Health Genomics 13: 492-494.

4. de Vries J, Tindana P, Littler K, Ramsay M, Rotimi C, Abayomi A, Mulder N, Mayosi BM, 2015. The H3Africa policy framework: negotiating fairness in genomics. Trends Genet 31: 117-119.

5. Mensah GA, Peprah EK, Sampson UK, Cooper RS, 2015. H3Africa comes of age. Cardiovasc J Afr 26 (2 Suppl 1): S3-S5.

6. Ramsay M, 2015. Growing genomic research on the African continent: the H3Africa Consortium. S Afr Med J 105: 10161017.

7. Ramsay M, Sankoh O, 2016. African partnerships through the H3Africa Consortium bring a genomic dimension to longitudinal population studies on the continent. Int $J$ Epidemiol 45: 305-308.

8. Wonkam A, Makani J, Ofori-Aquah S, Nnodu OE, Treadwell M, Royal C, Ohene-Frempong K; Members of the H3Africa Consortium, 2015. Sickle cell disease and H3Africa: enhancing genomic research on cardiovascular diseases in African patients. Cardiovasc J Afr 26 (2 Suppl 1): S50-S55.

9. Mulder N, Abimiku A, Adebamowo SN, de Vries J, Matimba A, Olowoyo P, Ramsay M, Skelton M, Stein DJ, 2018. H3Africa: current perspectives. Pharmacogenomics Pers Med 11:59-66.

10. Tom Kariuki, Simon Kay, 2017. There Are Not Enough Scientists in Africa. How Can We Turn This Around? World Economic Forum on Africa, Available at: https://www.weforum.org/agenda/ 2017/05/scientists-are-the-key-to-africas-future/. Accessed May 31, 2019.

11. Sirugo $\mathrm{G}$ et al., 2010. Report on the 6th African Society of Human Genetics (AfSHG) meeting. March 12-15, 2009, Yaoundé, Cameroon. Am J Trop Med Hyg 83: 226-229.

12. Gurdasani $D$ et al., 2015. The African Genome Variation Project shapes medical genetics in Africa. Nature 517: 327-332. 